AperTO - Archivio Istituzionale Open Access dell'Università di Torino

\title{
Molecular and Histological Changes in Post-Treatment Biopsies of Non-Squamous Non-Small Cell Lung Cancer: A Retrospective Study
}

\section{This is the author's manuscript}

Original Citation:

\section{Availability:}

This version is available http://hdl.handle.net/2318/1596236

since 2016-11-08T22:55:23Z

Published version:

DOI:10.1007/s11523-015-0383-8

Terms of use:

Open Access

Anyone can freely access the full text of works made available as "Open Access". Works made available under a Creative Commons license can be used according to the terms and conditions of said license. Use of all other works requires consent of the right holder (author or publisher) if not exempted from copyright protection by the applicable law. 


\section{MOLECULAR AND HISTOLOGICAL CHANGES IN POST-TREATMENT BIOPSIES OF NON -}

SQUAMOUS NON-SMALL CELL LUNG CANCER: A RETROSPECTIVE STUDY

Vatrano $\mathrm{S}^{*}$, Righi $\mathrm{L}^{*}$, Vavalá $\mathrm{T}^{* *}$, Rapa $\mathrm{I}^{*}$, Busso $\mathrm{M}^{* * *}$, Izzo $\mathrm{S}^{*}$, Cappia $\mathrm{S}^{*}$, Veltri $\mathrm{A}^{* * *}$, Papotti M*, Scagliotti GV**, Novello S**.

Divisions of * Pathology, ** Medical Oncology and *** Radiology, Department of Oncology, University of Turin at San Luigi Hospital, Orbassano (Torino), Italy

\section{Corresponding author:}

Mauro Papotti, MD, Department of Oncology, University of Turin, San Luigi Hospital, Regione Gonzole 10, 10043 Orbassano (Torino), Italy

E-mail : mauro.papotti@unito.it

Phone :+390119026276

Fax : +390119026753

Short title: Re-biopsy in non-squamous NSCLC 
Conflict of interest: A.V.: honoraria from Cook Medical, Astrazeneca, Amgen, Eli Lilly, Roche, Mundipharma, Bristol Myers Squibb, Novartis; M.P.: honoraria from Eli Lilly, Novartis, Pfizer, Clovis Oncology; G.V.S.: honoraria from Eli Lilly, Astra Zeneca, Pfizer, Roche, Clovis Oncology, Icon; S.N.: honoraria from Eli Lilly, Boehringer Ingelheim, Roche, Astra Zeneca, MSD. S.V., L.R., T.V., I.R., M.B., S.I. and S.C. have no conflicts of interest to declare. 


\section{ABSTRACT}

Background: Recently, in advanced non-small cell lung cancer (NSCLC), standard chemotherapy was flanked by biological agents directed against genomic abnormalities, including EGFR and ALK alterations, that significantly improved patients' outcome. Despite these achievements, tumor progression almost always occurs and a reassessment of the tumor genetic profile may contribute to modulate the therapeutic regimen. A re-sampling may provide tissue for additional tests to detect acquired resistance and/or new genetic alterations, but the currently available information is limited.

Patients and Methods: Histological and genetic re-assessments of bioptical or surgical tissue samples from 50 non-squamous NSCLC patients before and after at least one systemic treatment were performed. EGFR, KRAS, BRAF, PIK3CA and HER2 mutations were sequenced, p.T790M was identified with Real-Time PCR and ALK and MET genomic alterations by Fluorescent In Situ Hybridization.

Results: Overall in baseline biopsies $37 / 50$ (74\%) tumors had genetic alterations, either single (52\%) or multiple (22\%). Among them, 16 were EGFR mutations and 6 ALK rearrangements. In the second tissue sampling, 54\% of cases had additional genomic changes, including newly acquired alterations (81\%) or losses (18\%). The commonest changes were MET amplification and p.T790M mutation. One case had an histological shift from adenocarcinoma to small cell carcinoma.

Conclusions: the remarkable number of molecular changes following systemic therapy and the genetic complexity of some cases underline the value of histological and molecular reevaluation of lung cancer to tailor the most appropriate therapy along disease progression.

KEY WORDS: Non Small Cell Lung Cancer, biopsy, molecular alteration, histology, re-biopsy, systemic therapy 


\section{INTRODUCTION}

Non-Small Cell Lung Cancer (NSCLC) accounts for $80 \%$ of lung cancer diagnoses, and approximately $70 \%$ of patients present at baseline with unresectable locally advanced or metastatic disease. In this setting, standard chemotherapy significantly improves patients' outcome, but 2- and 5-year survival remains disappointing [1]. From 2004 and onward genetic alterations have been identified, mainly in the adenocarcinoma subtype, such as sensitizing mutations of the Epidermal Growth Factor Receptor (EGFR) and rearrangements of the Anaplastic Lymphoma Kinase (ALK) genes and specific agents directed against these genomic abnormalities significantly extended progression-free survival of treated patients [2-4].

Historically, these targeted therapies had been offered to patients with peculiar clinical characteristics (never smokers, females with adenocarcinoma histology) and in this subgroup of patients a second biopsy was more frequently performed. More recently, based on the outcome of phase III studies performed in genotype-defined patients [5-7], the systematic search for EGFR sensitizing mutations and ALK re-arrangements has been routinely implemented, to better understand acquired resistance mechanisms.

Acquired resistance is usually multifactorial and reflects tumor heterogeneity, whereby resistance clones already exist in the tumor at diagnosis and become dominant as drug sensitive cells die or, alternatively, tumors develop an adaptive response with prevailing anti-apoptotic signals. The knowledge on these changes is almost entirely limited to EGFR mutation-positive NSCLCs that harbor the resistance mutation p.Thr790Met (p.T790M) in exon 20, a finding reported at the time of disease progression in approximately $50 \%$ of the cases. More recently, in ALK rearranged tumors, several ALK kinase domain mutations have been identified with only a slight preponderance of the gatekeeper mutation L1196M [8]. No information is currently available about mutational profile changes in patients harboring other genetic abnormalities and/or treated with cytotoxic chemotherapy alone. 
The diagnostic attitude to perform a re-biopsy at the time of disease progression, although strongly recommended in the context of clinical trials, is not routine practice because of the lack of effective therapeutic treatments once a specific molecular abnormality is detected.

In the present study we performed histological and genetic re-assessments of tumor tissues from 50 patients with non-squamous NSCLC sampled before and after at least one systemic treatment, in order to determine the potential impact of pathological and genetic changes in rebiopsy specimens.

\section{MATERIALS AND METHODS}

\section{Case series}

Fifty patients with a pathologic diagnosis of non-squamous NSCLC and treated the San Luigi Hospital, University of Turin, from July 2006 to February 2014 were retrospectively selected from pathology archives and considered for the study if formalin fixed paraffin embedded (FFPE) tumour samples obtained at baseline and at the end of the front-line treatment or the time of disease progression were available. The search of EGFR sensitivity mutations and ALK translocations was performed at the time of the original diagnosis in 28 and 9 cases, respectively.

Patients were grouped into two subgroups: one $(n=31)$ consisting of stage IV tumours and another $(n=19)$ with early $(n=5)$ or locally advanced $(n=14)$ disease (Table 1$)$. Metastatic patients received systemic treatments (cytotoxic chemotherapy or targeted therapies). Conversely, in the other group, one patient received surgery alone, four received surgery followed by adjuvant chemotherapy, 10 received neo-adjuvant chemotherapy followed by surgery and four sequential chemo- and radiotherapy with curative intent. Follow-up data were available for all patients. 


\section{DNA extraction and mutational analysis}

One of the pathologists (LR) re-assessed each tumour sample for adequacy (set at a minimum of $30 \%$ of tumor cells [9]) according to current recommendations [10].

Genomic DNA from FFPE tissue samples was extracted and purified using QIAmp DNA FFPE Tissue kit (Qiagen, Hilden - Germany), according to the manufacturer's instructions and quantified by spectrophotometry (Eppendorf, Hamburg, Germany), as previously described [9]. All 100 specimens yielded DNA content and quality adequate for mutational testing. Mutational analyses of EGFR (NM_005228) in exon 18 (codon 719 variants), exon 21 (codons 858 and 861 variants) and exon 19 (from codons 746 to 750) were performed using EGFR TKI response (sensitivity) kit (CE-IVD, Diatech, Jesi - Italy)[9]. Furthermore, $100 \mathrm{ng}$ of genomic DNA were used to amplify target regions containing mutational hotspots of KRAS exon 2 (NM_4985.3), PIK3CA exon 10 and 21 (NM_6218.2), HER2 exon 20 (NM_4448.2), ALK exon 22 and 23 (NM_004304) [two genetic changes known to be associated with resistance to crizotinib], and BRAF exon 11 and 15 (NM_004333.4) genes, by means of real-time end-point PCR using a Rotor-Gene $Q$ instrument (Qiagen, Hilden - Germany). The presence of PCR products for each amplimer was detected by Melting-analysis with a denaturation step from $65^{\circ} \mathrm{C}$ up to $95^{\circ} \mathrm{C}$. Direct sequencing was performed for mutational analysis of genes investigated using PyroMark Q96 machine (Biotage, Uppsala - Sweden). Pyrosequencing results were further validated by allelic-specific PCR (ARMS) of EGFR exons 18,19 and 21, as indicated by guidelines [10] and previously described [9].

All samples were also tested for the presence of the p.T790M mutation in EGFR exon 20 by ARMS, using specific primers. PCR reaction was performed in a $25 \mu$ volume mixture containing 2X Master mix (Promega, Madison - USA), 20X EVA Green dye (Biotium, Hayward, CA - USA), 0.4 $\mu \mathrm{M}$ of each primer and $50 \mathrm{ng}$ template. The amplification was carried out under the following condition: $95^{\circ} \mathrm{C}$ for 5 minutes, then 40 cycles of $95^{\circ} \mathrm{C}$ for 40 seconds, $57.6^{\circ} \mathrm{C}$ for 30 seconds and 
$72^{\circ} \mathrm{C}$ for 40 seconds and a final extension step of $72^{\circ} \mathrm{C}$ for 5 minutes, on Rotor-Gene $Q$ (Qiagen Hilden, Germany) instrument. Amplification was detected by melting analysis (ramping range of $65^{\circ} \mathrm{C}-95^{\circ} \mathrm{C}$ and rising steps of 1 degree), corresponding to the presence of the mutation investigated. According to the guidelines [10], genomic DNA (gDNA) reference standard (Horizon diagnostics, Cambridge, United Kingdom) harbouring the p.T790M mutation at $1.25 \%$ of mutated allele was used as positive control, whereas gDNA reference standard wild type acted as negative control.

\section{Gene rearrangement/amplification assessment by Fluorescent In Situ Hybridization (FISH)}

FISH assay to detect ALK rearrangements and MET amplification were performed on $5 \mu \mathrm{m}$ unstained FFPE tumour tissue sections using the ALK break-apart probe set (Vysis LSI ALK Dual Color break-apart rearrangement probe; Abbott Molecular, USA) and MET probe set (Vysis MET SpectrumRed FISH Probe Kit; Abbott Molecular, USA), according to the manufacturers' instructions. Briefly, the slides were de-paraffinized and quenched in 1x saline-sodium citrate (SSC) buffer at $98^{\circ} \mathrm{C}$ for 15 minutes, followed by protease digestion with a ready to use protease $\mathrm{K}$ solution $(0.25 \mathrm{mg} / \mathrm{ml}$, Invitrogen Life Technologies, USA). The ALK and MET probes were hybridized at $37^{\circ} \mathrm{C}$ for 16 hours after co-denaturing at $73^{\circ} \mathrm{C}$ for 3 minutes. Stringency wash was conducted at $73^{\circ} \mathrm{C}$ with 2 X SSC-0.3\%NP40 for 2 minutes. The slides were dehydrated and counterstained with 4,6-diamidino-2-phenylindole (DAPI) II with the anti-fade compound $p$ phenylenediamine. Slides were evaluated with a triple-pass filter microscope (DAPI, Orange, Green) under a 100x objective lens in oil immersion. ALK FISH-positive cases were defined as those having more than $15 \%$ of nuclei with split signals (isolated red and green signals with a distance corresponding at least to twice the largest signal size) or with a fusion signal and one red signal alone. 
All cases with mean gene copy number $\geq 5$ were considered MET FISH-positive according to Cappuzzo et al [11]. Furthermore, the presence of polisomy, both high and low, was evaluated following the UCCC (University of Colorado Cancer Center) criteria [12] and considered MET FISH-negative.

\section{Statistical analyses}

Categorical variables were reported using frequency distribution and summarized as means and range. Association between categorical variables were assessed using Chi-square or Fisher's exact test. Overall survival was calculated from the time of diagnosis to death or last follow-up. The log-rank test was used to assess differences between groups. Significant $p$ value was set at p<0.05; statistical GraphPad software (PRISM 5, San Diego, CA) was used.

\section{RESULTS}

\section{Patients' characteristics}

Clinical-pathological characteristics of the series are summarized in Table 1. For a long period of time (2004-2010) at our hospital genomic analyses were driven by clinical enrichment criteria and this diagnostic strategy explains the median age of 54 years (range 26-72) and the minority of males (40\%), being 21 patients never smokers. The initial biopsy was performed on the primary lung lesion in 31 out of 50 patients (62\%), while in $19(38 \%)$ the tumour sample was obtained from metastatic deposits ( 8 extra-mediastinal lymph nodes, 4 mediastinal lymph nodes, 4 pleura, 1 brain, 1 liver, 1 skin). Diagnosis of adenocarcinoma (ADC) was made in 40/50 (80\%) patients (including 2 ADC with an associated sarcomatoid component) and in the remaining $10(20 \%)$ cases, the diagnosis was NSCLC. Among such 10 cases, the single case in the metastatic group was further immunoprofiled and a diagnosis of "NSCLC, favour ADC" was 
rendered. All the others belonged to the early/locally advanced group and were not further subtyped because the histotype was assessed in the surgical specimens.

Among the 31 metastatic patients, EGFR mutation test at the time of the original diagnosis was performed in 23 cases ( 7 cases were positive, all treated with EGFR-TKIs) and ALK FISH test in 9 ( 3 positive, all treated with crizotinib). All the other patients underwent chemotherapy.

Among the 19 patients with early/locally advanced disease, the routine genomic analyses (EGFR mutations and ALK translocation) were less often requested (chi square $p=0.026$ for both genomic markers when compared to the metastatic group).

The re-biopsy was performed after a mean time of 17 months (range 3-58) from baseline diagnosis (16 months for both the metastatic and early/locally advanced groups), and tissue specimens were obtained from lung primaries in 29 out of 50 (58\%) tumours, while 21/50 (42\%) were from metastatic sites. After front line systemic treatment, all but one ADC diagnoses were confirmed, and in this latter case a morphological switch to small cell lung cancer (SCLC) with neuroendocrine features (synaptophysin positive) was detected (Figure 1). In those nine early/locally advanced cases diagnosed as NSCLC-NOS at baseline, at the time of the reevaluation, the surgical samples revealed 6 ADC, 1 pleomorphic sarcomatoid carcinoma, 1 large cell neuroendocrine carcinoma and 1 adenosquamous carcinoma. In the five cases with stage I/II disease at diagnosis, radiological and endo-bronchial findings at relapse fully supported the recurrence of the baseline disease and did not indicate the presence of a second primary lung cancer.

Mean follow-up time was of 37 months (range 4-151); in detail, 8/19 (42\%) early/locally advanced and 19/31 (61\%) metastatic patients died, with median overall survival (OS) of 57 (range 4-151) and 36 (range 8-65) months (CI 95\%, 0,19-1,07), respectively.

\section{Molecular profiling at baseline}


The complete molecular profiling is outlined in Figure 2 and summarized in Table 2. Tumour samples harboured either single or multiple genomic changes (at least two genetic alterations in the same or in different genes). Twenty-six samples (52\%, 17 metastatic, 9 early/locally advanced) had only one genomic abnormality (9 EGFR, 5 ALK, 3 MET, 6 KRAS, 2 p.T790M and 1 PIK3CA) and 11 (22\%, 7 metastatic, 4 early/locally advanced) had two genetic alterations (7 EGFR with 3 MET, 3 p.T790M and one KRAS; 1 ALK with pT790M; 1 MET with PIK3CA and 2 KRAS with p.T790M). The remaining 13 cases ( 7 in the metastatic and 6 in locally advanced group) were wild type for all tested genetic alterations.

Finally, the biopsy sampling site (primary or metastases) of the tumor at the two sampling time (either the same or different) did not influence the event of genomic changes identified in the two patients group (Table 2, lower half).

Molecular profiling of tumours in the re-biopsy at the time of tumour progression or surgery following chemotherapy

At the re-biopsy (Figure 2), 22 cases (46\%, 13 metastatic, 9 early/locally advanced) had one genomic change ( 1 actEGFR, 5 ALK, 6 MET, 6 KRAS, 2 pT790M; 1 PIK3CA and 1 BRAF), while 20 (38\%, 12 metastatic, 8 early/locally advanced) had more than one genomic change (15 with two and 5 with three genetic alterations). Among 13 wild type tumour samples at baseline, eight acquired genomic changes in MET (5 cases), exon 20 EGFR (1 case), BRAF (1 case) and KRAS (2 cases) genes. Overall, a higher level of genetic heterogeneity was observed, specifically, in 27 out of 50 (54\%, 16 metastatic and 11 early/locally advanced) cases either new $(22 / 27,81 \%)$ or lost $(5 / 27,18 \%)$ genetic alterations were detected. Among such cases, no new EGFR sensitizing mutations were acquired de novo along disease progression, whereas they were maintained in 14 out of $16(87 \%)$ positive cases at baseline, being two EGFR mutations lost in exon 19 . At the 
re-biopsy, p.T790M point mutation was lost in 2 out of 5 cases and acquired in other 4 tumour samples.

In the early/locally advanced group p.T790M mutation and MET amplification showed the higher amounts of changes: p.T790M mutation was lost in one case and acquired in 4 cases while MET amplification was acquired in 7 cases and maintained in 3 cases. KRAS and PIK3CA were documented in one case each.

The single case of adenocarcinoma with a histotype switch to SCLC at re-biopsy (after six cycles of cytotoxic cisplatin and pemetrexed-based chemotherapy; patient \#14 of Figure 2a) showed EGFR p.L858R and p.T790M point mutations at baseline but the loss of the p.T790M mutation in the second biopsy (Figure 1).

\section{Genetic and clinical-pathological correlations}

Either in early/locally advanced or in metastatic tumors the genotype (including any type of EGFR mutations, ALK, MET, PI3K and KRAS) was not associated with smoking habits, or any other of the considered clinical and pathological variables (including sex, age and clinical stage in early/locally advanced cancers, therapy, type and site of samples).

When molecular profiles were dichotomized between those remained unchanged and those modified at the re-biopsy in both metastatic and early/locally advanced tumours, none of the considered clinical or pathological parameters listed above showed a statistical association. Only a trend was observed for the distribution of sensitizing EGFR mutations between metastatic and early/locally advanced groups at the first biopsy $(p=0.06)$, but not in re-biopsy samples. Moreover, considering correlations between genomic changes and differences in postprogression survival, only the presence of MET amplification in the re-biopsy sample showed a trend to poor prognosis (hazard ratio: $0.34, \mathrm{Cl} 95 \%$ 0.09-1.3; $\mathrm{p}=0.06$ ) in the metastatic group 
(data not shown) whereas no differences in post-progression survival were observed when considering all the other genomic changes.

\section{DISCUSSION}

We retrospectively re-assessed the molecular profiling in selected genes of 50 cases of nonsquamous NSCLC that had paired tumour samplings, at the time of diagnosis and of disease progression (or at the time of surgery), as available in the pathology archive of our Department. The panel of investigated genes was quite comparable to that investigated by the Lung Cancer Mutation Consortium [13] and in a French National Study [14]. The incidence of mutations in these two large studies and in our case-series cannot be compared for several reasons including our limited sample size and the influence of selection factors (i.e. clinical enrichment criteria mainly for EGFR mutation and ALK translocation) that in our centre drove the clinical decision of performing a second biopsy. This explains why $44 \%$ of the cases harboured "druggable" molecular alterations (actEGFR and ALK), whereas 30\% of cases showed mutations in genes usually associated to acquired resistance to targeted therapies (namely, MET, KRAS, EGFR_p.T790M and PIK3CA [13]). These data support the hypothesis of a primary mechanism of resistance, such as EGFR p.T790M mutation and MET gene copy number alteration, in tumour specimens of patients with no prior exposure to EGFR TKI [15]. In our series, the prevalence of MET FISH positive cases at baseline (14\%) was in line with Cappuzzo et al. [11] (11.1\%), but higher than values reported by others [16] (see below).

The presence of primary resistance alterations has also been significantly associated with poorer outcome to specific TKI therapies $[17,18]$, but it is not definitively proven yet if the presence of these genomic changes influence the therapeutic choice. Recently, the CAP/IASLC/AMP guidelines mandated testing for EGFR mutations and ALK fusions to guide therapy selection with an EGFR or ALK inhibitor, respectively, in all patients with advanced-stage 
adenocarcinoma, regardless of sex, race, smoking history, or other clinical risk factors, and to prioritize EGFR and ALK testing over other molecular predictive tests. However, in the daily clinical practice and because of past onsite unavailability of molecular testing for some time, as for instance in our series, the primary driver for testing was the never-smoking status. This minimalistic approach definitively underestimates the incidence of oncogene addicted lung cancer at baseline. In our series, 9 out of 22 tumour samples positive for EGFR sensitizing mutations or ALK translocation were detected in former/current smokers. However, the molecular analyses in these nine samples were anyhow lately performed and all the patients received the appropriate therapy, as second or third line, during the course of the disease. In fact, in our series, only 8 patients received matched therapy in the metastatic group (respectively 5 gefitinib and 3 crizotinib alone), while none in the early/locally advanced group in the absence of a regulatory indicators for targeted therapies in this disease stages.

The post-treatment assessment of additional tumour tissue revealed several molecular changes (either losses or acquisitions) in $54 \%$ of cases (27/50) compared to the baseline profile, and a change in the histological subtype in $2 \%(1 / 50)$ of cases. Among the 13 cases wild type at baseline, 8 tumours showed either druggable or resistance-related mutations in the re-biopsy samples. The limited set of genes investigated is not representative enough of the complexity of the acquired resistance mechanisms, but further genomic investigations were not feasible because of the limited amounts of tumor tissue available. This further stresses on the one hand the relevance of adequately triaging tissue samples for both diagnostic and molecular purposes, and, on the other, the need of high throughput technologies for comprehensive molecular profiling. This is even more relevant in the advanced disease setting, where limited tissue samples are usually available. 
Beyond technical aspects the need for re-biopsy to assess the histological and molecular tumour profile of non-squamous NSCLC after treatment and/or disease progression is a matter of debate in the perspective of treatment personalization.

While the assumption of the mutual exclusivity between exon 19-21 EGFR and KRAS mutation and ALK translocation was quite evident at baseline similarly to what reported by others [19], the co-existence of two of these three genetic changes increased at the time of the re-biopsy. The most frequently acquired genetic alterations were EGFR point mutation p.T790M and MET amplification, in line with the reported main mechanisms of acquired resistance $[15,20]$, irrespective of the type of therapy administered.

In this specific context, even if retrospective and in a small and selected number of patients, some unique findings can be extrapolated from the group of tumours with early or locally advanced stage that were mainly treated with cytotoxic chemotherapy. In such group, excluding the cases with oncogene addiction (3 EGFR mutations and one ALK translocation), acquired MET amplification was observed in 7 out of 15 cases. These data differ from literature data and the discrepancy could reflect the highly conflicting results about MET positivity prevalence in NSCLC, ranging from $2 \%$ to $20 \%$, probably due to different methods of analysis and criteria used for FISH scoring [21,22]. Cappuzzo et al [11], investigated a series of radically resected NSCLC and reported a MET amplification in approximately $11.1 \%$ of the cases. In other studies, the positive rate was much lower (3\%), but the definition of MET highly amplified tumours was limited to those exceeding the mean of 6 gene copy number per cell $[16,23]$. However, in one of these studies, the Authors also found an additional $30 \%$ of cases with MET intermediate or low altered signals; both squamous and non-squamous treatment-naive NSCLC showed different extents of MET copy number gains of the intermediate or low type, according to the interpretation criteria used [16], supporting the concept of a complex array of MET changes. The clinical significance of these changes is far from being understood, at least in the 
intermediate/borderline subgroup, including the role of MET amplification in the crosstalk between MET/HGF axis and $\beta$-catenin signalling, among other pathways [24].

Concerning EGFR mutations, the p.T790M point mutation was lost in 4 out of 8 cases positive at baseline and was acquired in other 8 cases. The significance of these findings remains largely undefined, with a reduced sensitivity to EGFR-TKI therapy in EGFR p.T790M mutated patients at baseline as opposed to a relative indolent disease course when the p.T790M is detected at progression [25]. Among other genetic alterations lost between baseline tumour sampling and the re-biopsy, one actEGFR mutations was lost probably due to a clonal selection induced by therapy, as previously proposed by Nguyen [25], while another patient had a loss of KRAS mutation probably related to the tumour heterogeneity of the different sampling sites (primary versus metastatic tissue).

The mechanisms leading to genomic heterogeneity among samples before and after treatment are still poorly understood. Both tumour heterogeneity and drug-induced selection mechanisms have to be taken into account and probably concur. The comparative analysis of clinical and pathological parameters among stable and unstable genotypes in our series failed to detect any significant correlation, including type of sample (thus excluding technical artefacts due to the amount of tumour cells available), type of therapy and site of the lesion sampled.

In our series, one case (2\%) underwent an histological shift to SCLC, followed by a further subsequent sarcomatoid transformation in a third biopsy (data not shown). Previous case reports described such histological transition in EGFR mutated lung cancers after therapy as a possible mechanism of drug resistance [26-28]. The molecular bases of SCLC transformation are not clearly understood. The cancer stem cell hypothesis could explain the existence of a pluripotent population of EGFR mutant cancer stem cells that could favour the development of an undifferentiated tumour cell population $[29,30]$. In our case of SCLC transformation, the p.T790M mutation was not detected. In a recent sequencing profiling of SCLC [31], only one 
case showed the p.T790M mutation, thus confirming the rare occurrence of this resistance mutation in such an histological type known to be resistant to the EGFR targeted therapy. In conclusion, despite the retrospective nature of the study and the small and selected number of patients tested, both the observed (54\%) remarkable fraction of additional molecular changes between baseline tumor biopsy and that following systemic therapy and the high genetic complexity of individual tumors underline the value of histological and molecular reevaluation of lung cancer, in order to tailor the most appropriate therapy along disease progression or subsequently to an initial systemic local treatment.

Acknowledgements: Work partially supported by a grant from the "Fondazione Guido Berlucchi", Brescia (approved April 24, 2013) to MP and HEALTH-F2- 2010-258677-CURELUNG from the European Community's Seventh Framework Program (FP7/2007-2013) to GVS.

SV is a PhD fellow at the University of Turin, Doctorate School of Biomedical Sciences and Oncology.

\section{REFERENCES}

1. Alberg AJ, Brock MV, Ford JG et al (2013) Epidemiology of lung cancer: Diagnosis and management of lung cancer, 3rd ed: American College of Chest Physicians evidence-based clinical practice guidelines. Chest 143 (5 Suppl):e1S-29S. doi:10.1378/chest.12-2345

2. Rosell R, Carcereny E, Gervais R et al (2012) Erlotinib versus standard chemotherapy as firstline treatment for European patients with advanced EGFR mutation-positive non-small-cell lung cancer (EURTAC): a multicentre, open-label, randomised phase 3 trial. Lancet Oncol 13 (3):239246. doi:S1470-2045(11)70393-X [pii] 10.1016/S1470-2045(11)70393-X

3. Mok T, Wu YL, Zhang L (2009) A small step towards personalized medicine for non-small cell lung cancer. Discov Med 8 (43):227-231 
4. Shaw AT, Kim DW, Nakagawa K et al (2013) Crizotinib versus chemotherapy in advanced ALKpositive lung cancer. N Engl J Med 368 (25):2385-2394. doi:10.1056/NEJMoa1214886

5. Inoue A, Kobayashi K, Maemondo M et al (2013) Updated overall survival results from a randomized phase III trial comparing gefitinib with carboplatin-paclitaxel for chemo-naive nonsmall cell lung cancer with sensitive EGFR gene mutations (NEJO02). Ann Oncol 24 (1):54-59. doi:mds214 [pii] 10.1093/annonc/mds214

6. Sequist LV, Yang JC, Yamamoto N et al (2013) Phase III study of afatinib or cisplatin plus pemetrexed in patients with metastatic lung adenocarcinoma with EGFR mutations. J Clin Oncol 31 (27):3327-3334. doi:JCO.2012.44.2806 [pii] 10.1200/JCO.2012.44.2806

7. Wu YL, Fukuoka M, Mok TS et al (2013) Tumor response and health-related quality of life in clinically selected patients from Asia with advanced non-small-cell lung cancer treated with firstline gefitinib: post hoc analyses from the IPASS study. Lung Cancer 81 (2):280-287. doi:S01695002(13)00109-8 [pii] 10.1016/j.lungcan.2013.03.004

8. Lovly CM, Pao W (2012) Escaping ALK inhibition: mechanisms of and strategies to overcome resistance. Sci Transl Med 4 (120):120ps2. doi:4/120/120ps2 [pii] 10.1126/scitranslmed.3003728 9. Righi L, Cuccurullo A, Vatrano S et al (2013) Detection and characterization of classical and "uncommon" exon 19 Epidermal Growth Factor Receptor mutations in lung cancer by pyrosequencing. BMC Cancer 13:114. doi:1471-2407-13-114 [pii] 10.1186/1471-2407-13-114 10. Lindeman NI, Cagle PT, Beasley MB et al (2013) Molecular testing guideline for selection of lung cancer patients for EGFR and ALK tyrosine kinase inhibitors: guideline from the College of American Pathologists, International Association for the Study of Lung Cancer, and Association for Molecular Pathology. J Thorac Oncol 8 (7):823-859. doi:10.1097/JTO.0b013e318290868f

11. Cappuzzo F, Marchetti A, Skokan M et al (2009) Increased MET gene copy number negatively affects survival of surgically resected non-small-cell lung cancer patients. J Clin Oncol 27 (10):1667-1674. doi:JCO.2008.19.1635 [pii] 10.1200/JCO.2008.19.1635 
12. Park S, Choi YL, Sung CO et al (2012) High MET copy number and MET overexpression: poor outcome in non-small cell lung cancer patients. Histol Histopathol 27 (2):197-207

13. Kris MG, Johnson BE, Berry LD et al (2014) Using multiplexed assays of oncogenic drivers in lung cancers to select targeted drugs. JAMA 311 (19):1998-2006. doi:1872815 [pii] 10.1001/jama.2014.3741

14. Barlesi F, Blons $H$, Beau-Faller $M$ et al Results of routine EGFR, HER2, KRAS, BRAF, PI3KCA mutations detection and EML4-ALK gene fusion assessment on the first 10,000 non-small cell lung cancer (NSCLC) patients (pts). In: ASCO, 2013. Journal of Clinical Oncology, p suppl. Abst 8000

15. Remon J, Moran T, Majem M et al (2014) Acquired resistance to epidermal growth factor receptor tyrosine kinase inhibitors in EGFR-mutant non-small cell lung cancer: a new era begins. Cancer Treat Rev 40 (1):93-101. doi:S0305-7372(13)00125-4 [pii] 10.1016/j.ctrv.2013.06.002

16. Schildhaus $H$, Schultheis AM, Ruschoff J et al (2014) MET amplification status in therapynaive adeno- and squamous cell carcinomas of the lung. Clin Cancer Res 15;21(4):907-15 doi:1078-0432.CCR-14-0450 [pii] 10.1158/1078-0432.CCR-14-0450

17. Rosell R, Molina MA, Costa C et al (2011) Pretreatment EGFR T790M mutation and BRCA1 mRNA expression in erlotinib-treated advanced non-small-cell lung cancer patients with EGFR mutations. Clin Cancer Res 17 (5):1160-1168. doi:1078-0432.CCR-10-2158 [pii] 10.1158/10780432.CCR-10-2158

18. Bean J, Brennan C, Shih JY et al (2007) MET amplification occurs with or without T790M mutations in EGFR mutant lung tumors with acquired resistance to gefitinib or erlotinib. Proc Natl Acad Sci U S A 104 (52):20932-20937. doi:0710370104 [pii] 10.1073/pnas.0710370104

19. Sequist LV, Waltman BA, Dias-Santagata D et al (2011) Genotypic and histological evolution of lung cancers acquiring resistance to EGFR inhibitors. Sci Transl Med 3 (75):75ra26. doi:10.1126/scitranslmed.3002003 
20. Minuti G, D'Incecco A, Landi L et al (2014) Protein kinase inhibitors to treat non-small-cell lung cancer. Expert Opin Pharmacother 15 (9):1203-1213. doi:10.1517/14656566.2014.909412

21. Guo B, Cen H, Tan X et al (2014) Prognostic value of MET gene copy number and protein expression in patients with surgically resected non-small cell lung cancer: a meta-analysis of published literatures. PLoS One 9 (6):e99399. doi:10.1371/journal.pone.0099399 PONE-D-1413031 [pii]

22. Gelsomino F, Facchinetti F, Haspinger ER et al (2014) Targeting the MET gene for the treatment of non-small-cell lung cancer. Crit Rev Oncol Hematol 89 (2):284-299. doi:S10408428(13)00253-9 [pii]10.1016/j.critrevonc.2013.11.006

23. Cancer Genome Atlas Research Network (2014) Comprehensive molecular profiling of lung adenocarcinoma. Nature 511:543-550

24. Gherardi E, Birchmeier W, Birchmeier C et al (2012) Targeting MET in cancer: rationale and progress. Nat Rev Cancer 12 (2):89-103. doi:nrc3205 [pii] 10.1038/nrc3205

25. Nguyen KS, Kobayashi S, Costa DB (2009) Acquired resistance to epidermal growth factor receptor tyrosine kinase inhibitors in non-small-cell lung cancers dependent on the epidermal growth factor receptor pathway. Clin Lung Cancer 10 (4):281-289. doi:10.3816/CLC.2009.n.039

26. Zakowski MF, Ladanyi M, Kris MG (2006) EGFR mutations in small-cell lung cancers in patients who have never smoked. N Engl J Med 355 (2):213-215. doi:355/2/213 [pii] 10.1056/NEJMc053610

27. Morinaga R, Okamoto I, Furuta K et al (2007) Sequential occurrence of non-small cell and small cell lung cancer with the same EGFR mutation. Lung Cancer 58 (3):411-413. doi:S01695002(07)00317-0 [pii] 10.1016/j.lungcan.2007.05.014

28. Fukui T, Tsuta K, Furuta K et al (2007) Epidermal growth factor receptor mutation status and clinicopathological features of combined small cell carcinoma with adenocarcinoma of the lung. Cancer Sci 98 (11):1714-1719. doi:CAS600 [pii]10.1111/j.1349-7006.2007.00600.x 
29. Visvader JE, Lindeman GJ (2008) Cancer stem cells in solid tumours: accumulating evidence and unresolved questions. Nat Rev Cancer 8 (10):755-768. doi:nrc2499 [pii] 10.1038/nrc2499 30. Rosen JM, Jordan CT (2009) The increasing complexity of the cancer stem cell paradigm. Science 324 (5935):1670-1673. doi:324/5935/1670 [pii] 10.1126/science.1171837

31. Ross JS, Wang K, Elkadi OR et al (2014) Next-generation sequencing reveals frequent consistent genomic alterations in small cell undifferentiated lung cancer. J Clin Pathol 67(9):7726. doi:jclinpath-2014-202447 [pii] 10.1136/jclinpath-2014-202447 


\section{FIGURE LEGENDS}

Figure 1: Histological shift from adenocarcinoma histology (a) to a morphological small cell carcinoma (b), expressing TTF1 and the neuroendocrine marker synaptophysin (inserts). (a, b: hematoxylin and eosin, 200X; inserts: 3,3'-Diaminobenzidine stain, 100X).

Figure 2: Genomic profiles at baseline and re-evaluation biopsies of patients with metastatic (a) or early/locally advanced (b) disease

Abbreviations: ND: not done; D: done; ADC: adenocarcinoma; NSCLC: non small cell lung cancer; NOS: not otherwise specified; SCLC: small cell lung cancer; Fav: favoured; SARC: sarcomatoid; ADSQC: adenosquamous carcinoma; LCNEC: large cell neuroendocrine carcinoma; CDDP: Cisplatin; Pem: Pemetrexed; Pazo: Pazopanib; Gef: Gefitinib; Crizo: Crizotinib; Gem: Gemcitabine; CBP: Carboplatin; Adv: adjiuvant; Neoadv: neo-adjiuvant; Surg: surgery; CarboPac: Carboplatin and Paclitaxel; ASA404: Vadimezan; Doce: Docetaxel; RT: radiotherapy; WT: wilde type; MUT: mutated; Alt(A): alterated with amplification; Alt(R): alterated with rearrangement. 
Table 1: Clinico-pathological characteristics of the case series

\begin{tabular}{|c|c|c|c|c|}
\hline \multicolumn{2}{|l|}{ Characteristics } & $\begin{array}{l}\text { Total } \\
(n=50)\end{array}$ & $\begin{array}{l}\text { Metastatic } \\
\text { Group } \\
(n=31)\end{array}$ & $\begin{array}{l}\text { Early/locally } \\
\text { advanced group } \\
\qquad(n=19)\end{array}$ \\
\hline \multicolumn{2}{|l|}{$\begin{array}{l}\text { Sex: } \\
\text { - Male }\end{array}$} & $\begin{array}{l}20(40 \%) \\
30(60 \%)\end{array}$ & $\begin{array}{l}10(32 \%) \\
21(68 \%)\end{array}$ & $\begin{array}{c}10(53 \%) \\
9(47 \%)\end{array}$ \\
\hline \multicolumn{2}{|c|}{ Median Age (range) } & $54(26-72)$ & $54(26-67)$ & $53(38-72)$ \\
\hline $\begin{array}{l}\text { Smoking status } \\
\text { - Never smoker } \\
\text { - Former/curren }\end{array}$ & & $\begin{array}{l}21(42 \%) \\
29(58 \%)\end{array}$ & $\begin{array}{l}15(48 \%) \\
16(52 \%)\end{array}$ & $\begin{array}{c}6(32 \%) \\
13(68 \%)\end{array}$ \\
\hline \multicolumn{2}{|l|}{$P S=0$} & $50(100 \%)$ & $31(100 \%)$ & 19 (100\%) \\
\hline \multirow{4}{*}{$\begin{array}{l}\text { Clinical stage at } \\
\text { diagnosis }\end{array}$} & I & 2 (4\%) & // & $2(11 \%)$ \\
\hline & II & $3(6 \%)$ & // & $3(16 \%)$ \\
\hline & III & $14(28 \%)$ & $/ /$ & $14(73 \%)$ \\
\hline & IV & $31(62 \%)$ & $31(100 \%)$ & $/ /$ \\
\hline \multicolumn{2}{|c|}{$\begin{array}{l}\text { Histology at the first biopsy } \\
\text { - ADC } \\
\text {-Other* }\end{array}$} & $\begin{array}{l}40(80 \%) \\
10(20 \%)\end{array}$ & $\begin{array}{c}30(97 \%) \\
1(3 \%)\end{array}$ & $\begin{array}{c}10(53 \%) \\
9(47 \%)\end{array}$ \\
\hline $\begin{array}{l}\text { Histology at the } \\
\text { - ADC } \\
\text {-Other** }\end{array}$ & jiopsy & $\begin{array}{c}46(92 \%) \\
4(8 \%)\end{array}$ & $\begin{array}{c}30(97 \%) \\
1(3 \%)\end{array}$ & $\begin{array}{c}16(84 \%) \\
3(16 \%)\end{array}$ \\
\hline $\begin{array}{l}\text { EGFR mutations } \\
\text { the first biopsy: }\end{array}$ & the time of & $28(56 \%)$ & $23(74 \%)$ & $5(26 \%)$ \\
\hline $\begin{array}{l}\text { ALK FISH tested } \\
\text { biopsy: }\end{array}$ & ne of the first & $9(18 \%)$ & $9(29 \%)$ & 0 \\
\hline
\end{tabular}

Abbreviations: PS: Performance Status; EGFR: Epidermal Growth Factor Receptor; ALK: Anaplastic Lymphoma Kinase; FISH: Fluorescent In Situ Hybridization; ADC: Adenocarcinoma;

*1 non small cell lung cancer favour ADC and 9 non small cell lung cancers.

**1 small cell lung carcinoma, 1 sarcomatoid (pleomorphic) carcinoma, 1 large cell neuroendocrine carcinoma, 1 adenosquamous carcinoma. 
Table 2: Summary of overall molecular alterations identified for each gene investigated at baseline and at the rebiopsy, according to patients groups.

\begin{tabular}{|c|c|c|c|c|c|}
\hline & \multicolumn{2}{|c|}{ METASTATIC GROUP (\#31) } & \multicolumn{2}{|c|}{$\begin{array}{l}\text { EARLY/LOCALLY ADVANCED } \\
\text { GROUP (\#19) }\end{array}$} \\
\hline \multirow{10}{*}{$\begin{array}{l}\text { NUMBER OF } \\
\text { CASES (more } \\
\text { than one gene } \\
\text { alteration could be } \\
\text { associated in the } \\
\text { same case) }\end{array}$} & & BIO1 & $\mathrm{BIO2}$ & BIO1 & $\mathrm{BlO2}$ \\
\hline & $\begin{array}{c}\text { EGFR } \\
\text { (ex 19-21) }\end{array}$ & $\begin{array}{c}13(3 \text { ex21+10 } \\
\text { ex19) }\end{array}$ & $\begin{array}{c}11 \text { (3 ex21+8 } \\
\text { ex19) }\end{array}$ & $\begin{array}{c}3(2 \text { ex21+1 } \\
\text { ex19) }\end{array}$ & $\begin{array}{c}3(2 \text { ex21+1 } \\
\text { ex19) }\end{array}$ \\
\hline & $\begin{array}{c}\text { EGFR } \\
\text { p.T790M } \\
\end{array}$ & 5 & 7 & 3 & 5 \\
\hline & ALK & 5 & 7 & 1 & 1 \\
\hline & MET & 4 & 7 & 3 & 10 \\
\hline & KRAS & 4 & 5 & 5 & 6 \\
\hline & PIK3CA & 0 & 1 & 2 & 3 \\
\hline & BRAF & 0 & 1 & 0 & 0 \\
\hline & HER2 & 0 & 0 & 0 & 0 \\
\hline & WT & 7 & $6(2)^{*}$ & 6 & $2(1)^{*}$ \\
\hline \multirow{3}{*}{$\begin{array}{l}\text { RELATIONSHIP } \\
\text { BETWEEN } \\
\text { GENOTYPE } \\
\text { AND SITE OF } \\
\text { BIOPSY } \\
\end{array}$} & & SAME SITE & $\begin{array}{l}\text { DIFFERENT } \\
\text { SITE }\end{array}$ & SAME SITE & $\begin{array}{l}\text { DIFFERENT } \\
\text { SITE }\end{array}$ \\
\hline & UNCHANGED & 10 & 5 & 2 & 6 \\
\hline & MODIFIED & 9 & 7 & 6 & 5 \\
\hline
\end{tabular}

* parentheses indicate number of cases previously mutated and reverted to WT (see text for details)

Abbreviations: EGFR: Epidermal Growth Factor Receptor; ALK: Anaplastic Lymphoma Kinase; MET: hepatocyte growth factor receptor; PIK3CA: phosphatidylinositol-4,5-bisphosphate 3kinase, catalytic subunit alpha; KRAS: Kirsten rat sarcoma viral oncogene homolog; BRAF: v-raf murine sarcoma viral oncogene homolog B1; HER2: human epidermal growth factor receptor 2; BIO1: first biopsy; BIO2: second biopsy; WT: Wild-Type; ex: exon. 


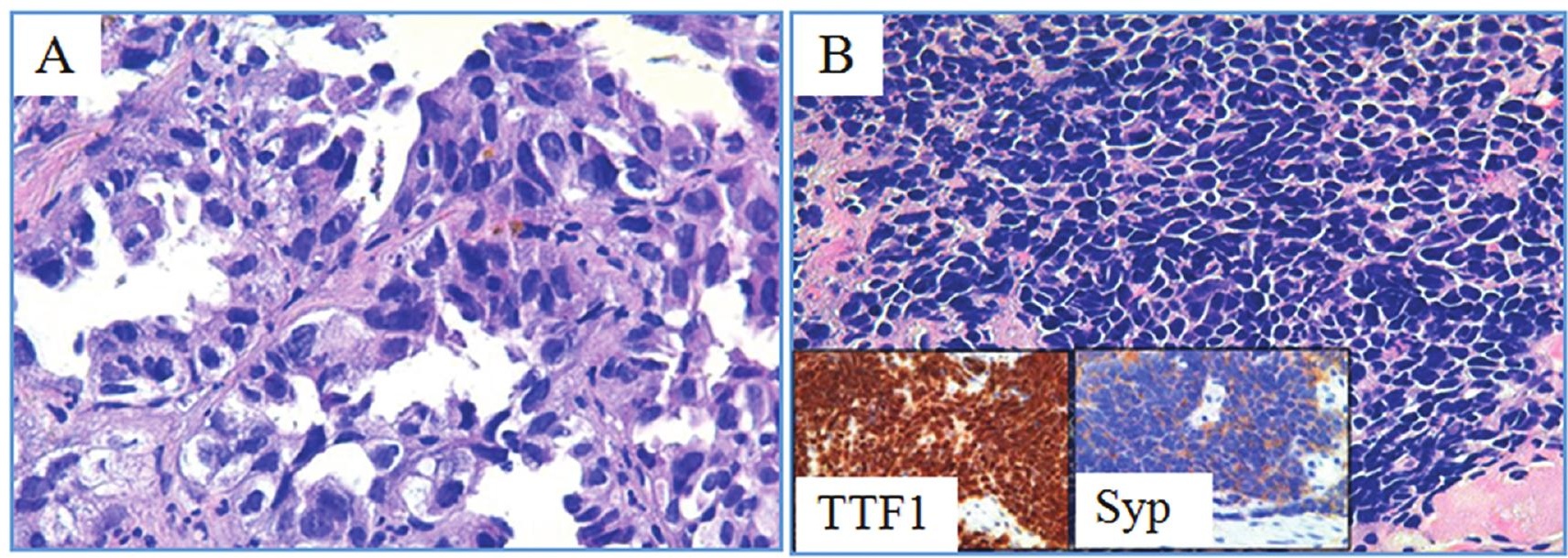


